\title{
Atomic Force Microscope Observation of Growth and Defects on As-Grown (111) 3C-SiC Mesa Surfaces
}

Philip G. Neudeck, Andrew J. Trunek ${ }^{1}$, and J. Anthony Powell ${ }^{2}$

NASA Glenn Research Center,

21000 Brookpark Road, M.S. 77-1, Cleveland, OH 44135, USA

${ }^{1}$ OAI, NASA Glenn, 21000 Brookpark Road, M.S. 77-1, Cleveland, OH 44135, USA

${ }^{2}$ Sest, Inc., NASA Glenn, 21000 Brookpark Road, M.S. 77-1, Cleveland, OH 44135 USA

\begin{abstract}
This paper presents experimental atomic force microscope (AFM) observations of the surface morphology of as-grown (111) silicon-face 3C-SiC mesa heterofilms. Wide variations in 3C surface step structure are observed as a function of film growth conditions and film defect content. The vast majority of as-grown $3 \mathrm{C}$-SiC surfaces consisted of trains of single bilayer height $(0.25 \mathrm{~nm})$ steps. Macrostep formation (i.e., step-bunching) was rarely observed, and then only on mesa heterofilms with extended crystal defects. As supersaturation is lowered by decreasing precursor concentration, terrace nucleation on the top (111) surface becomes suppressed, sometimes enabling the formation of thin $3 \mathrm{C}-\mathrm{SiC}$ film surfaces completely free of steps. For thicker films, propagation of steps inward from mesa edges is sometimes observed, suggesting that enlarging $3 \mathrm{C}$ mesa sidewall facets begin to play an increasingly important role in film growth. The AFM observation of stacking faults (SF's) and $0.25 \mathrm{~nm}$ Burgers vector screw component growth spirals on the as-grown surface of defective $3 \mathrm{C}$ films is reported.
\end{abstract}

\section{INTRODUCTION}

The growth of high-quality 3C-SiC heteroepitaxial films on top of arrays of mesas etched into commercial on-axis wafers has recently been reported [1-5]. In particular, growth by the "step-free surface heteroepitaxy" technique has achieved 3C-SiC mesa films entirely free of stacking fault (SF) and double-positioning boundary (DPB) defects on the majority of $4 \mathrm{H} / 6 \mathrm{H}-$ $\mathrm{SiC}$ mesas that are free of substrate screw dislocations (SD's). However, only two initial AFM scans of as-grown $3 \mathrm{C}$ mesa film surfaces have been published to date [5]. This paper presents more extensive AFM observations of as-grown (111) 3C-SiC mesa heterofilm surfaces.

\section{EXPERIMENTAL}

The experimental processes for achieving and characterizing high-quality $3 \mathrm{C}-\mathrm{SiC}$ heterofilm growth (including polytype confirmation by multiple independent techniques) on top of arrays of mesas patterned into commercial $4 \mathrm{H} / 6 \mathrm{H}$ on-axis wafers have been detailed previously [1-5]. Table I describes specific growth process variations applied to the samples reported in this paper. The $4 \mathrm{H}-\mathrm{SiC}$ substrates with patterned mesas were placed into a horizontal flow epitaxial reactor and subjected to a pre-growth high temperature etch in either $\mathrm{HCl}$ or $\mathrm{H}_{2}$. It should be noted that the uncoated graphite in the reactor supplies additional carbon to the growth environment, as we have observed $\mathrm{SiC}$ growth even when propane flow is reduced to zero. The top surfaces of mesas that were free of SD's were then rendered completely free of atomic scale steps by carrying out 
homoepitaxial growth at a temperature $\left(\mathrm{T}_{\text {step-free }}\right)$ [6]. The temperature was then ramped down over a period of time $\left(\mathrm{t}_{\mathrm{ramp}}\right)$ to the 3C-SiC nucleation temperature $\left(\mathrm{T}_{3 \mathrm{C} \text {-nucleate }}\right)$ to intentionally nucleate $3 \mathrm{C}-\mathrm{SiC}$ on the large step-free (0001) $4 \mathrm{H}$ surface [1-5]. At the conclusion of the rampdown, the temperature was set to $\mathrm{T}_{3 \mathrm{CG}}$ for a time period $\left(\mathrm{t}_{3 \mathrm{CG}}\right)$ until the end of the growth run. Following growth, the reactants were shut off and temperature ramped down in hydrogen.

Four consecutive $3 \mathrm{C}-\mathrm{SiC}$ runs (2A-D in Table I) were carried out on the same wafer (Sample 2 ) at nearly the same $3 \mathrm{C}$ growth temperature $\left(\mathrm{T}_{3 \mathrm{CG}}\right)$. Runs $2 \mathrm{~A}$ and $2 \mathrm{C}$ were conducted under "higher supersaturation" growth conditions induced by higher flows of silane and propane. Runs 2B and 2D were "lower supersaturation" in that silane and propane flows were halved (Table I). Because the $3 \mathrm{C}$ film was already established for mesas on this sample by run $2 \mathrm{~A}$, Runs $2 \mathrm{~B}-\mathrm{D}$ consisted of only a pre-growth hydrogen etch followed by temperature rampdown and resumption of $3 \mathrm{C}$ film growth. Over a dozen SD-free mesas on Sample 2 were characterized by AFM following each of the growths. All AFM scans were obtained by tapping mode [7]. Some AFM images shown below were digitally altered to erase bad scan lines and particulate contamination.

\section{RESULTS AND DISCUSSION}

\section{AFM Detection of Stacking Faults (Sample 1)}

We have previously reported that SD's threading a $4 \mathrm{H}-\mathrm{SiC}$ substrate mesa subsequently result in a defective $3 \mathrm{C}$ heterofilm, and that the presence of SF defects can greatly enhance $3 \mathrm{C}$ heterofilm growth rate [1-3,5]. Fig. 1a shows an AFM of a small region of the as-grown epitaxial surface of a defective $3 \mathrm{C}-\mathrm{SiC}$ film grown on a $4 \mathrm{H}$ mesa threaded by a SD on Sample 1. A pattern of $0.25 \mathrm{~nm}$ height steps generally flows from the upper left corner with downstep direction toward the lower right of the Fig. 1a AFM image. Superimposed on the $0.25 \mathrm{~nm}$ step pattern are a series of line features (some denoted by arrows in Fig. 1a), most of which are sub- $0.25 \mathrm{~nm}$ (i.e., sub-bilayer) height perturbations in the surface. Fig. 1b shows an optical micrograph of the same region following over 5 hours of dry thermal oxidation. The dark lines visible in Fig. $1 \mathrm{~b}$ are well known to arise from enhanced oxidation that occurs where SF's intersect the surface of a 3C-SiC film [8]. Given the exact correspondence of the pattern of linear features in Figs. 1a and 1b, we conclude that SF's can be observed by AFM on as-grown 3C-SiC films. SF features were also observed by AFM on additional samples, including some Sample 2 mesas.

The vast majority of steps observed by AFM on as-grown $3 \mathrm{C}$ surfaces were $0.25 \mathrm{~nm}$ (i.e., a single Si-C bilayer) in height. Macrosteps were occasionally observed on mesas with SF defects. Most macrosteps formed when $0.25 \mathrm{~nm} 3 \mathrm{C}$ growth steps piled-up along a SF. An example of such pileup can be seen in Fig. 3b of [5]. However, most SF's, including those shown in Fig. 1, did not produce macrosteps. Because growth steps in Fig. 1a flow across the SF's, instead of 

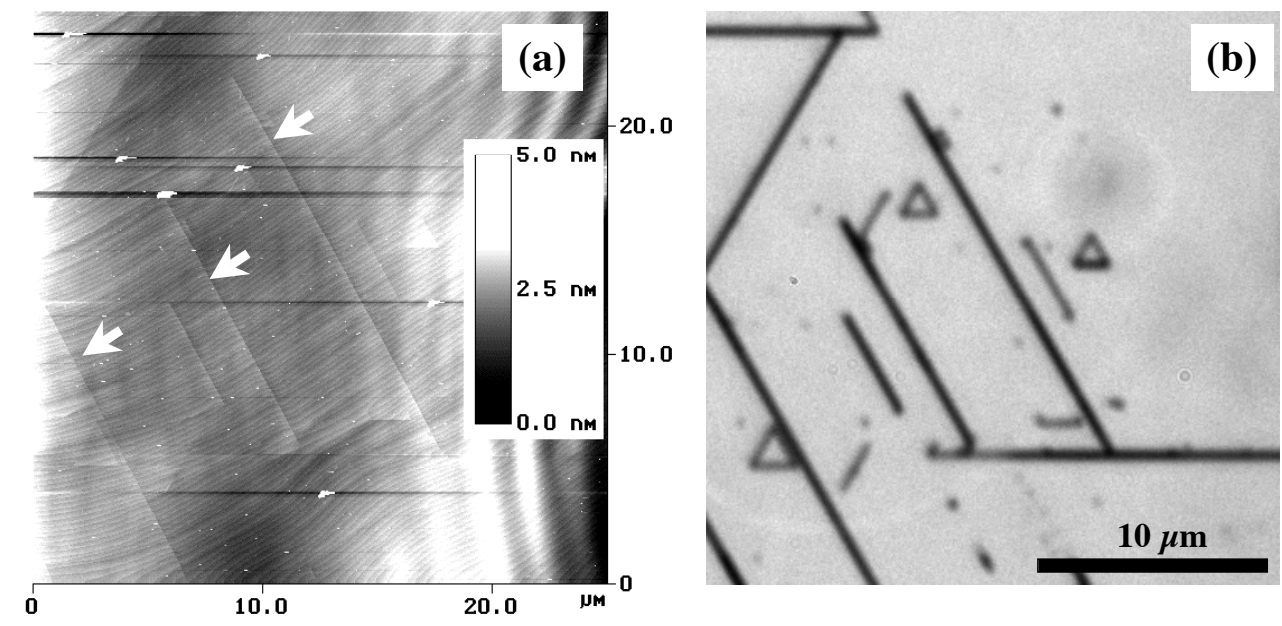

Fig. 1: Stacking faults on a defectively grown 3C-SiC heterofilm surface revealed by (a) AFM of as-grown surface and (b) post-oxidation optical micrograph.

emanating outward from the SF's, we conclude that the faults shown in Fig. 1 are not a primary nucleation source of new bilayers on this $3 \mathrm{C}$-SiC mesa surface. However, it is worth noting that we have observed enhanced inward growth of new bilayers from mesa edges for some instances where SF's intersect the sidewall edge of a 3C mesa [5]. Given the variety of behaviors noted above, further studies are clearly necessary for better understanding of SF's in the 3C-SiC film growth process. It is important to note that the majority of 3C-SiC heterofilms grown by stepfree surface heteroepitaxy (on SD-free 4H/6H mesas) are free of SF-defects [1-4].

\section{C Surfaces as Function of Growth Conditions and non-SF Defect Content (Sample 2)}

AFM measurements revealed significant differences in step structure between growth under higher and lower supersaturation conditions. Fig. 2 shows two examples of step structure observed on nearby mesas (Mesa A and Mesa B) after higher supersaturation Run 2A. Both step patterns have very rough edges and are generally concentric with the topmost terrace residing in the interior of the mesa shape. However, Mesa B clearly shows a much higher $(\sim 10 \mathrm{X})$ step density than Mesa A.
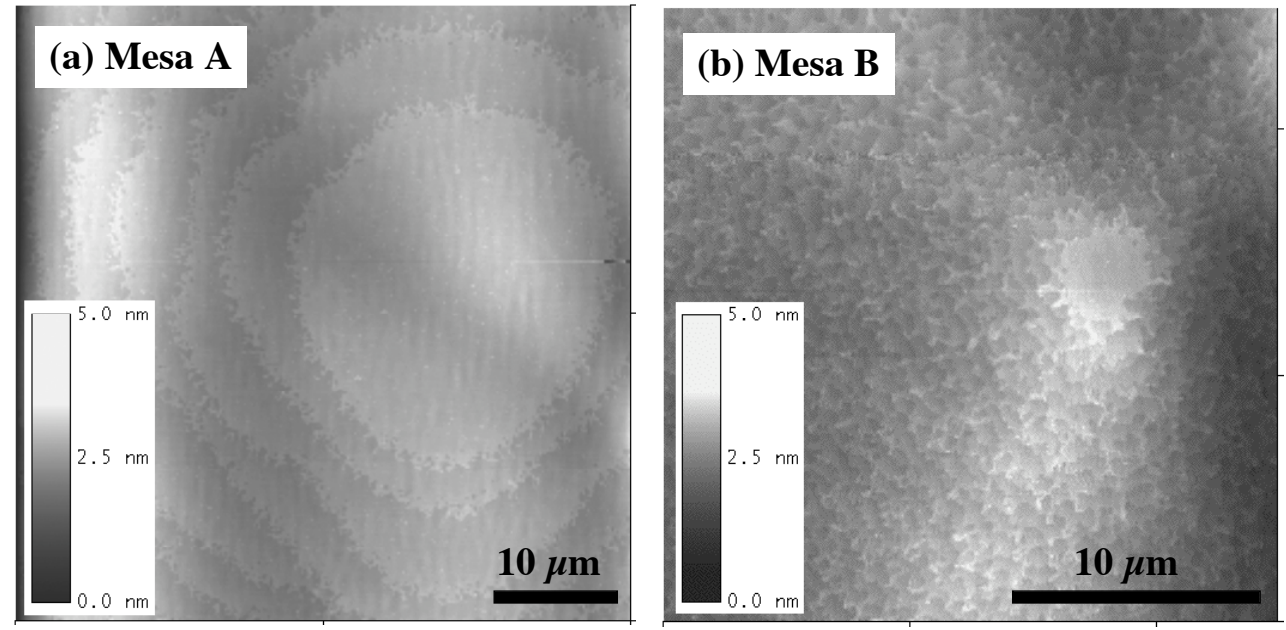

Fig. 2: AFM scans showing the topmost terraces from nearby (a) Mesa A and (b) Mesa B on the same wafer following higher supersaturation growth Run 2A. Both step patterns consist of concentric steps with jagged edges, but the step density is close to $10 \mathrm{X}$ greater for Mesa B. 

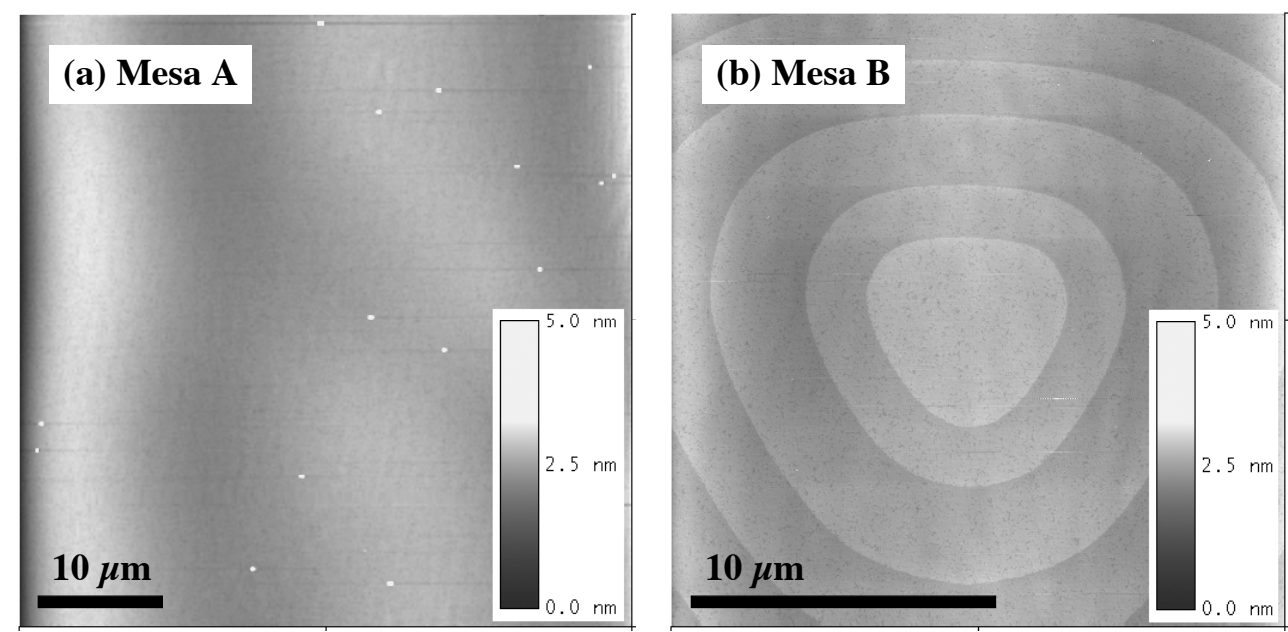

Fig. 3: AFM scans showing the same Mesa A and Mesa B of Fig. 2 following lower supersaturation growth Run 2B. No steps were detected anywhere on Mesa A. The concentric step pattern of Mesa B is consistent with bilayer growth by defect-assisted terrace nucleation.

Fig. 3 shows AFM scans of the same mesas following a short-duration low supersaturation growth Run 2B. As shown in Fig. 3a, no surface steps were detected on Mesa A. Thus, terrace nucleation was effectively suppressed, enabling the first reported formation of a step-free $3 \mathrm{C}-\mathrm{SiC}$ mesa surface. In contrast, Mesa B (Fig. 3b) shows a well-ordered concentric pattern of $0.25 \mathrm{~nm}$ high steps. The concentric step pattern and higher step density of Fig. $3 \mathrm{~b}$ is consistent with our previous observations of defect-assisted terrace nucleation on a very thin $3 \mathrm{C}$-SiC growth surface [5]. We hypothesized that dislocations (threading edge) lower terrace nucleation energy leading to the observed concentric step pattern, but transmission electron microscopy (TEM) studies are not yet complete [4,5]. Most of the mesas observed at this stage were step-free (Fig. 3a), or exhibited far lower step density than shown in Fig. $3 b$.

Following substantial additional growth under higher supersaturation conditions (Run 2C), a rough step structure was again observed (Fig. 4). Under higher supersaturation conditions, nuclei of larger size are more likely to form on terraces prior to joining the main stepfronts [9]. The higher reactant concentration should facilitate more rapid stepflow expansion of existing nuclei and steps. These factors would favor a much rougher observed "non-equilibrium" stepfront expansion, consistent with our observations. Growth at lower supersaturation, where terrace nucleation is effectively suppressed, maintains a much smoother "equilibrium" stepfront. Under these conditions, we surmise that mobile surface adatoms are able to diffuse to fill kink sites in the slower expanding stepfront, thereby enabling the smooth stepfront structure.

In a significant number of cases after Run $2 \mathrm{C}$, the top of the step pattern was observed to originate from mesa edge regions. Fig. 4a (Mesa C) shows one example of this behavior. The observed trend for new bilayers to proceed inward from mesa edges on thicker $3 \mathrm{C}$ films suggests that enlarging 3C mesa sidewall facets may increasingly impact the evolution of thicker 3C crystals. An examination of the crystal structure of 3C-SiC reveals that all mesa sidwall/edge facets will have a higher percentage of surface carbon atoms than (the $0 \%$ of) the (111) siliconface of the mesa top surface. The reported nucleation probability of carbon-terminated $\mathrm{SiC}$ surfaces is substantially higher than for silicon-terminated surfaces [9]. Therefore, we propose that growth of new 3C crystal material (i.e., nucleation of new bilayers) on mesa edge/sidewall facets should occur more readily than on the Si-face top surface. As $3 \mathrm{C}$ mesa film thickness increases, the area of $3 \mathrm{C}$ edge/sidewall facets increases. Thus, we believe that it is possible that 

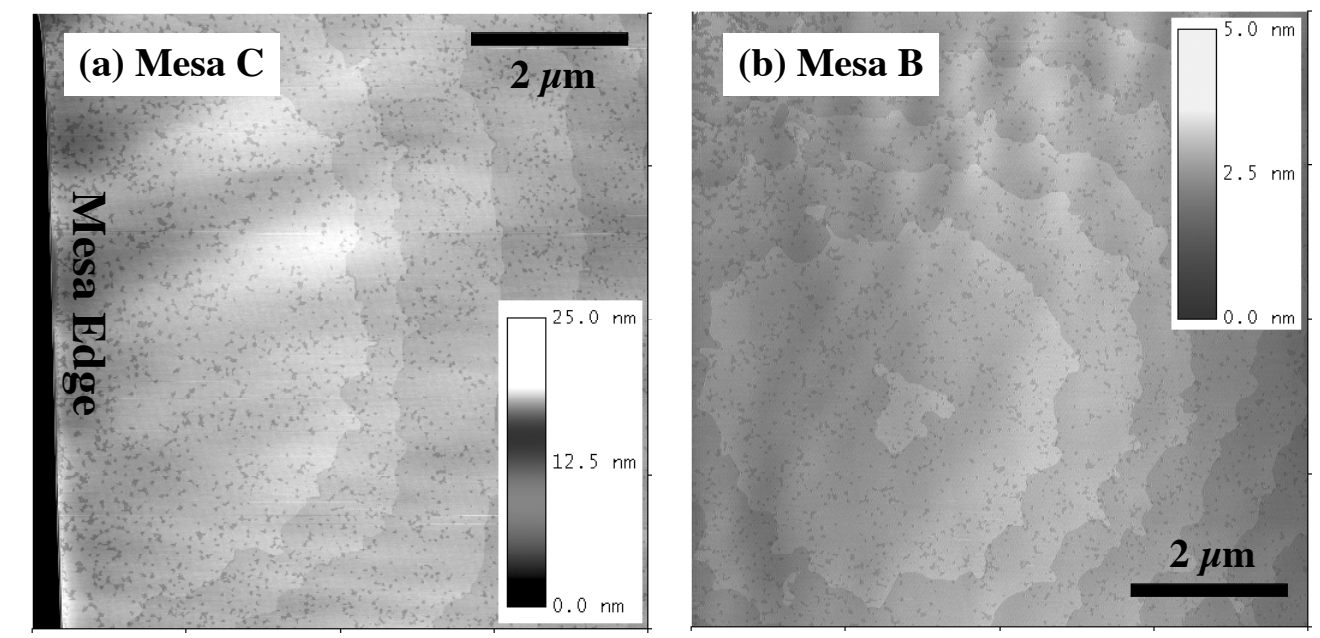

Fig 4: AFM scans showing the topmost terraces from two nearby mesas following higher supersaturation growth Run $2 \mathrm{C}$. The topmost terrace shown in (a) on Mesa C emanates from the side edge of the mesa, whereas the topmost terrace shown in (b) on Mesa B arises in the interior presumably due to defect-assisted nucleation.

mesa edge/sidewall facet growth may begin to impact evolution of thicker $3 \mathrm{C}$ mesa crystals, even when edge/sidewall facets have much smaller surface areas than the mesa top. Further studies are needed to experimentally verify or refute the proposed edge/sidewall growth model. In other cases, nucleation (and therefore the topmost observed terrace) takes place in the interior region of the mesa top surface. Fig. 4b shows the topmost terrace of Mesa B, so we surmise that nucleation is defect-assisted in this case.

Fig. 5 shows two examples of the topmost terrace regions on mesa surfaces following the final short-duration lower supersaturation growth (Run 2D). Quite interestingly, Fig. 5a reveals that bilayer height $(0.25 \mathrm{~nm})$ triangular-shaped stepfronts originate at a spiral stepsource. By analogy to spiral growth observed at screw dislocations in $4 \mathrm{H}-\mathrm{SiC}[3,6,10]$, we surmise that either a pure screw or mixed dislocation has formed in the 3C-SiC film. From the spiral, we
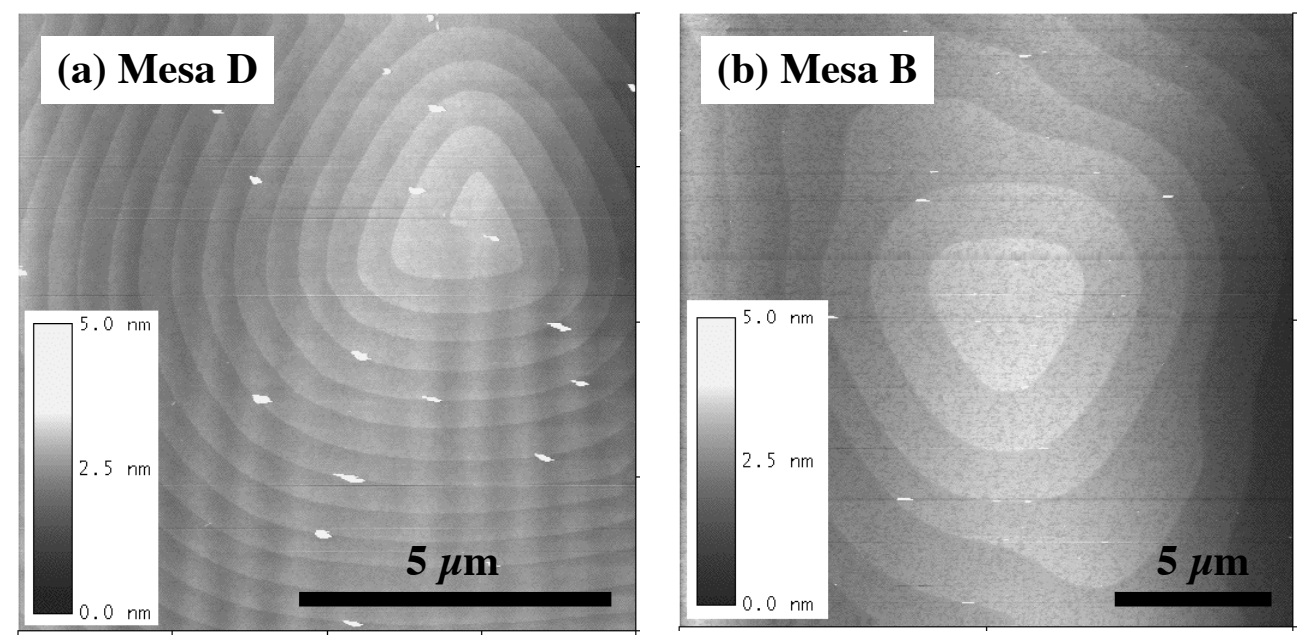

Fig. 5: AFM scans showing the topmost terraces from two mesas following lower supersaturation growth 2D. $0.25 \mathrm{~nm}$ height steps provided by (a) spiral stepsource from dislocation with $1 / 3$ c lattice parameter screw component, and (b) defect assisted terrace nucleation on the same Mesa B from Figs. 2-4b. 
deduce that the Burgers vector of the axial screw component of the dislocation is $0.25 \mathrm{~nm}, 1 / 3$ the $0.75 \mathrm{~nm} \mathrm{c}$-axis repeat distance of the $3 \mathrm{C}$ polytype [10]. We believe that this is the first published report of such a step structure observed on 3C-SiC. Because a screw dislocation is a continuous kinetic source of new growth steps, terrace nucleation is not required to support growth of thicker films. Therefore, the observed 3C-SiC step density and vertical growth rate should be highest for the spiral case, compared to growth where new 3C-SiC bilayers must first be initiated by thermodynamic terrace nucleation [5]. Indeed, the lateral density of bilayer height steps shown on Mesa B (Fig. 5b), grown by defect-assisted nucleation, is more than 3X smaller than the kinetic step density evolved from the spiral on Mesa D in Fig. 5a. The formation mechanism for the 3C-SiC spiral defect is unknown at this time.

\section{CONCLUSION}

This paper has presented a variety of AFM observations of the surface step structure of (111) 3C-SiC mesa heterofilm surfaces. The high quality of the $3 \mathrm{C}$ films enabled direct AFM observation of individual defects on the growth surface. The observed step patterns indicate that quite different mechanisms can govern the growth of adjacent $3 \mathrm{C}$ mesas on a given sample. The observed patterns provide evidence that film growth conditions, film defect content, and film thickness impact the evolution of 3C mesa crystals.

\section{ACKNOWLEDGEMENTS}

The authors would like to gratefully acknowledge the valuable assistance of Michelle Mrdenovich, Emye Benavage, Beth Osborn, Charles Blaha, John Heisler, Kimala Walker, Mike Artale, Pete Lampard, Glenn Beheim, Gary Hunter and Lawrence Matus at NASA Glenn Research Center. This work was funded by NASA Glenn Research Center under the Ultra Efficient Engine Technology, Advanced Electrical Components, and Revolutionary Aeroproplusion Concepts and programs.

\section{REFERENCES}

1. P. G. Neudeck, et al., Mat. Sci. Forum 389-393, 311-314 (2002).

2. P. G. Neudeck, et al., Mat. Sci. Forum 433-436, 213-216 (2003).

3. P. G. Neudeck and J. A. Powell, "Homoepitaxial and Heteroepitaxial Growth on Step-Free SiC Mesas," Silicon Carbide: Recent Major Advances, ed. W. J. Choyke, H. Matsunami, and G. Pensl, (Springer-Verlag, 2003) pp. 179-205.

4. P. Neudeck, J. A. Powell, A. Trunek, D. Spry, Mat. Sci. Forum 457-460, 169-174 (2004).

5. A. Trunek, P. Neudeck, J. A. Powell, D. Spry, Mat. Sci. Forum 457-460, 261-264 (2004).

6. J. A. Powell et al., Appl. Phys. Lett. 77, 1449-1451 (2000).

7. Veeco Digital Instruments, Dimension 3000, http://www.veeco.com.

8. J. A. Powell et al., Appl. Phys. Lett. 59, 183-185 (1991).

9. T. Kimoto and H. Matsunami, J. Appl. Phys. 75, 850-859 (1994).

10. J. A. Powell and D. J. Larkin, Phys. Status Solidi B 202, 529-548 (1997). 